\title{
ALKBH5-Mediated M6a-Demethylation Of USP1 Regulated T-Cell Acute Lymphoblastic Leukemia Cell Glucocorticoid Resistance by Stabilizing Aurora B
}

\section{Hongtao Gong}

The Second Affiliated Hospital of Zhengzhou University

Liu Liu ( $D$ liuliu1983w@163.com )

The First Affiliated Hospital of Zhengzhou University https://orcid.org/0000-0002-1204-1235

\section{Lina Cui}

The First Affiliated Hospital of Zhengzhou University

\section{Hongyan Ma}

The Second Affiliated Hospital of Zhengzhou University

\section{Liyun Shen}

The Second Affiliated Hospital of Zhengzhou University

\section{Research Article}

Keywords: Ubiquitin-specific protease 1, Aurora B, N6-methyladenosine demethylase, chemoresistance, Tcell acute lymphoblastic leukemia

Posted Date: March 31st, 2021

DOl: https://doi.org/10.21203/rs.3.rs-368055/v1

License: (1) This work is licensed under a Creative Commons Attribution 4.0 International License. Read Full License 


\section{Abstract}

Recent studies have evidenced that ubiquitin-specific proteases (USPs) are associated with the occurrence and drug resistance of T-cell acute lymphoblastic leukemia (T-ALL). N6-methyladenosine (m6A) demethylase AlkB homolog 5 (ALKBH5) exerts a carcinogenic effect in human cancers and improves the mRNA stability of USPs. Whether ubiquitin specific protease 1 (USP1) controls chemoresistance in T-ALL is unknown. Our study demonstrated that USP1, Aurora kinase B (Aurora B) and ALKBH5 levels were highly expressed in glucocorticoid (GC)-resistant T-ALL patients and cells (CEMC1). High expression of USP1 was correlated to the poor prognosis of T-ALL patients. Silencing USP1 increased CEM-C1 cell sensitivity to dexamethasone (Dex), reduced cell invasion, promoted cell apoptosis, and ameliorated glucocorticoid receptor (GR) expression. USP1 mediated T-ALL chemoresistance by interacting with Aurora B. Overexpression of USP1 reversed the promotive effects of Aurora B inhibitor on the sensitivity of CEM-C1 cells to Dex, cell apoptosis and GR level and the inhibition effect on cell invasion. Downregulation of ALKBH5 reduced the levels of USP1 and Aurora B, facilitated CEM-C1 cell sensitivity to Dex, apoptosis and GR expression, suppressed cell invasion. However, overexpression of USP1 reversed all the effects of ALKBH5 on CEM-C1 cells. In vivo results showed that tail vein injection of sh-USP1 resulted in a significant prolongation of mouse survival and maintained the normal weight of mice compared to the Dex group, reduced USP1 expression and facilitated GR expression. In conclusion, downregulation of USP1 ameliorated glucocorticoid resistance of T-cell acute lymphoblastic leukemia cells through suppressing Aurora B expression and elevating GR level.

\section{Introduction}

T-cell acute lymphoblastic leukemia (T-ALL) is an aggressive hematologic malignancies of $T$ cell progenitors, which accounts for $10-15 \%$ of acute lymphoblastic leukemia (ALL) cases in children and $25 \%$ of adults [1]. Although most chemotherapy regimens have high efficacy in pediatric patients, T-ALL has a high risk of recurrence owing to being prone to treatment resistance [2]. Chemoresistance is one of critical reasons preventing T-ALL from being cured. Hence, it is imperative to explore the resistance mechanism of T-ALL.

Ubiquitination is a highly conserved modification process, which has pivotal protein-regulatory functions and is critically regulated by deubiquitinating enzymes (DUBs) [3]. DUBs could be divided into five families, the largest of which is ubiquitin specific protease (USPs) [4]. Abnormal expression of DUBs is associated with many biological processes, including chemoresistance [5]. For example, USP2 was upregulated in triple-negative breast cancer (TNBC), and inhibition of USP2 enhanced TNBC responsiveness to chemotherapy [6]. High expression of USP20 promoted tumorigenesis and chemoresistance via regulating the deubiquitination of $\beta$-catenin [7]. USP9X accelerated breast cancer chemoresistance through reducing Yes-associated protein 1 (YAP1) polyubiquitination [8]. USP1 is a member of USP deubiquitylases subfamily, but its role in T-ALL chemoresistance remains unclear. 
$\mathrm{N}^{6}$-methyladenosine (m6A) RNA methylation is a reversible and the most predominant type mRNA modification [9]. The effects of m6A are achieved through the dynamic interaction of "writers" (methyltransferases), "erasers" (demethylases) and "readers" (effector proteins) [10]. The classical complex of writers comprises methyltransferase-like 3 (METTL3), methyltransferase-like 14 (METTL14) and Wilms tumor 1-associated protein (WTAP), which catalyze the formation of m6A [11]. AlkB homolog 5 (ALKBH5) and fat mass and obesity-associated (FTO) act as the erasers to reverse the m6A modification [9]. m6A modification is implicated in multiple biological processes including tumorigenesis. METTL3 facilitated tumor progression through an m6A-IGF2BP2-dependent mechanism in colorectal carcinoma [12]. FTO, acting as an oncogene, promoted breast tumor progression via repressing BNIP3 [13]. ALKBH5 maintained tumorigenicity of glioblastoma stem-like cells by enhancing FOXM1 expression and cell proliferation [14]. FTO promoted the growth of non-small cell lung cancer cells by increasing the expression of USP7 [15]. Nevertheless, the biological role of ALKBH5 related to m6A modification has not been fully explained in T-ALL.

In our study, we demonstrated that USP1 was upregulated in GC-resistant T-ALL patient bone marrow compared with GC-sensitive and normal patients, and high level of USP1 was associated with poorer survival in A-TLL patients. Mechanistically, we found that USP1 could regulate chemoresistance via interacting with Aurora B. Additionally, USP1 deubiquitinated and stabilized Aurora B to promote chemoresistance in CEM-C1 cells, suggesting that USP1 could be a promising prognostic marker and a potential therapeutic target in T-ALL.

\section{Material And Methods}

\section{Cell culture and treatments}

Bone marrow specimens from GC-sensitive patients ( 12 males and 8 females; average age 12 years old) and GC-resistant patients ( 9 males and 11 females; average age 15 years old) were obtained from A-TLL patients at the Department of Hematology, the Second Affiliated Hospital of Zhengzhou University from July 2016 to May 2019. This research was approved by the Medical Ethics Committee of the First Affiliated Hospital of Zhengzhou University. All A-TLL patients and healthy donors have got the written informed consent and were conducted in accordance with the Declaration of Helsinki. The diagnosis of ATLL was based on the revised French-American-British classification.

\section{Cell lines and culture}

GC-sensitive cell line (CEM-C7), GC-resistant cell line (CEM-C1) and HEK293T cells were obtained from Chinese Academy of Sciences (shanghai, China). All cells were maintained in Roswell Park Memorial Institute-1640 (RPMI-1640) medium (Gibco, Rockville, MD) supplemented with 10\% fetal bovine serum (FBS) (HyClone, Salt Lake City, UT) and 1\% penicillin-streptomycin (Sigma, St. Louis, MO, USA) at $37{ }^{\circ} \mathrm{C}$ in a controlled humidified atmosphere with $5 \% \mathrm{CO}_{2}$. Aurora B inhibitor barasertib (AZD1152-HQPA) was 
prepared in DMSO at the concentration of $5 \mathrm{mM}$ and stored at $-20^{\circ} \mathrm{C}$. Before co-culture with cells, the stock solution was diluted to a final concentration of $5 \mu \mathrm{M}$ with culture medium.

\section{Reverse transcription-quantitative PCR (RT-qPCR)}

Total RNA from bone marrow and cells was extracted using Trizol reagent (Invitrogen, Carlsbad, CA, USA), and all RNA was reversely transcribed into cDNA using PrimeScript RT reagent Kit (Takara, Dalian China) following the manufacturer's instructions. RT-qPCR were performed using SYBR Premix Ex Taq Kit (Takara, Dalian, China) on an Applied Biosystems Prism 7900 system (Applied Biosystems, Foster City, CA, USA). The PCR procedure was performed at $95^{\circ} \mathrm{C}$ for $30 \mathrm{~s}$, followed by 39 circles at $95{ }^{\circ} \mathrm{C}$ for $5 \mathrm{~s}$ and $60^{\circ} \mathrm{C}$ for $30 \mathrm{~s}$. GAPDH served as an internal reference. Data were analyzed using the $2^{-\triangle \triangle C T}$ method. The following primers were used: USP1 (forward 5'-3': AAA AGG GTG TGA GGC TCG AG; reverse 5'-3': CCA GGA AAA T CT GGT CGG CT); Aurora B (forward 5'-3': CTG CAC CAT CCC AAC ATC CT; reverse 5'-3': TGC CAA CTC CTC CAT GAT CG); ALKBH5 (forward 5'-3': CCA CCA GCT TTT GGA TCA CCA; reverse 5'-3': ACG CCT GGT TAC TAT CAA AAG G). Gene-Specific m6A qPCR was performed as previously reported by Li et al [16]. The housekeeping gene HPRT1 was used as internal control.

\section{Cell transfection}

The plasmids carrying USP1 expression kit and control vector were purchased from Sangon (Shanghai, China). Lipofectamine 3000 Transfection Reagent was used for cell transfection. After incubation, the reagent mixed with USP1 or vector was added in each group. In addition, siRNA negative control (scramble), USP1 siRNA, and ALKBH5 siRNA (All obtained from Sangon, Shanghai, China) were transfected into cells as described above.

\section{Cell viability}

CEM-C1 cells were seeded in 96-well plates overnight. After treatment with different concentration of Dex, $10 \mu \mathrm{L}$ of CCK-8 solution (Bio-Rad, Hercules, CA, USA) was added to each well for another $2 \mathrm{~h}$ at $37^{\circ} \mathrm{C}$. The absorption value was detected with a microplate reader (Molecular Devices, Sunnyvale, CA, USA) at the absorbance of $450 \mathrm{~nm}$.

\section{Cell invasion}

CEM-C1 cells $\left(2 \times 10^{4}\right.$ cells $/ 200 \mu \mathrm{L}$ of serum-free) were seeded into the upper chambers of Transwell chambers coated with Matrigel, and the lower chambers were filled with $600 \mu \mathrm{L}$ cell suspension. After incubation for $48 \mathrm{~h}$, cells attached to the upper surface of the filter membranes were removed with cotton swabs and the migrated cells were stained with $0.1 \%$ crystal violet for $20 \mathrm{~min}$. The number of migrated cells was quantified under a microscope (LEICA, shanghai, China).

\section{Cell apoptosis}


Cells were treated differently for $24 \mathrm{~h}$ and then were collected at a centrifuge of $300 \mathrm{~g}$ at $4{ }^{\circ} \mathrm{C}$ for $5 \mathrm{~min} .5$ $\mu \mathrm{L}$ of Annexin V-FITC and $5 \mu \mathrm{L}$ of propidium iodide (PI) were added at room temperature in dark and mixed gently for 20 min. After washing with binding buffer, cells were examined by Flow cytometry (BD Biosciences, San Jose, CA, USA).

\section{Co-Immunoprecipitation (Co-IP)}

After different transfection, HEK293T cells were collected and lysed using protein lysis buffer consisting of $50 \mathrm{mM}$ Tris- $\mathrm{HCl}, 150 \mathrm{mM} \mathrm{NaCl}, 1 \%$ Triton X-100, 1 mM EDTA, $200 \mathrm{mM}$ Na3VO4, and proteinase inhibitor. About $3 \mathrm{mg}$ of lysates were incubated with Flag-agarose beads or HA- agarose beads for $4 \mathrm{~h}$ at $4{ }^{\circ} \mathrm{C}$. Beads were washed four times with lysis buffer. $5 \mathrm{mg}$ of lysates were incubated with IgG (Millipore, Billerica, MA, USA) or anti-Aurora B for $4 \mathrm{~h}$ at $4^{\circ} \mathrm{C}$. Protein $\mathrm{A}$ agarose beads were added and incubated again at $4{ }^{\circ} \mathrm{C}$ for $12 \mathrm{~h}$. Lysis buffer was used to wash the immunocomplexes, eluted and boiled in SDS sample buffer. All samples were analyzed by Western blot with the indicated antibodies.

\section{Western blotting}

Cells were seeded in 6-well plates and treated differently. After lysing in Radio Immunoprecipitation Assay lysis buffer (Beyotime, Shanghai, China) for $30 \mathrm{~min} 4{ }^{\circ} \mathrm{C}$, protein concentration was quantified by using an Enhanced BCA Kit (Beyotime, Shanghai, China). Separated protein samples were transferred to polyvinylidene difluoride membranes (Millipore, Bedford, MA, USA) and blocked with 5\% skim milk at room temperature for $1 \mathrm{~h}$, and then the blots were incubated with primary antibodies against USP1 (ab108104, 1:400 dilution, Abcam, Cambridge, UK), Aurora B (ab2254, 1:500 dilution, Abcam, Cambridge, UK), GR (ab144525, 1:500 dilution, Abcam, Cambridge, UK), ALKBH5 (ab195377, 1:500 dilution, Abcam, Cambridge, UK). Then the membranes were incubated with a horseradish peroxidase-conjugated secondary antibody (1:1000 dilution, A32733, Abcam, Cambridge, MA, USA) for $1 \mathrm{~h}$ at room temperature. Chemiluminescent reagent was used to detect protein bands. All western blots were subjected to relative optical density (OD) analysis during the experiment, and signal was visualized using a chemiluminescence imaging system (Bioshine ChemiQ 4800 mini, China, Oxiang, Shanghai).

\section{Tumor xenograft experiments}

Adult male C57BL/6J mice (weighting 18-25 g) were obtained from Laboratory Animal Center, Zhengzhou University. Mice were randomly divided into four group: control group; control + Dex (mice were given with $8 \mathrm{mg} / \mathrm{kg}$ Dex); sh-RNA +Dex (mice were injected with $2 \mathrm{mg} / \mathrm{Kg}$ sh-RNA and given with $8 \mathrm{mg} / \mathrm{kg}$ Dex); shUSP1 +Dex (mice were injected with $2 \mathrm{mg} / \mathrm{Kg}$ sh-USP1 and given with $8 \mathrm{mg} / \mathrm{kg}$ Dex). After the treatment of each group, the mice were housed and fed in a room with an ambient temperature of $25^{\circ} \mathrm{C}$ for 80 days, and the survival time and weight of the mice was recorded. When rats were scarified, tissues were harvested for Western blotting. The study was approved by the ethic committee in the First Affiliated Hospital of Zhengzhou University. All animal experimental procedures were under the Guide for the Care and Use of Laboratory Animals of the National Institutes of Health. 


\section{Statistical Analysis}

Each experiment was carried out in triplicate, and all data were reported as the mean \pm standard error of mean (SEM). One-way analysis of variance was used for the comparison among groups, and $t$-test was used for pairwise comparison. ${ }^{\star} P \otimes 0.05$ was regarded significant.

\section{Results}

\section{USP1 mRNA and protein levels were upregulated in the bone marrow of T-ALL patients}

To explore the roles of USP1, qPCR was performed to detect the expression of USP1 in T-ALL patients. Compared with Normal bone marrow, the mRNA levels of USP1 were upregulated in GC-sensitivity and GC-resistant T-ALL patients (Fig.1 A, $P<0.05$ ). In relation to the GC-sensitivity group, USP1 expression was higher than that in GC-resistance group $(P<0.05)$. Additionally, survival analysis of T-ALL patients showed that patients with high expression of USP1 had poor overall survival (Fig.1B, $P<0.05$ ). Cell viability results showed that CEM-C1 cells had stronger tolerance to Dex than CEM-C7 cells (Fig. $1 \mathrm{C}$ and $\mathrm{D}$, $P<0.05)$. Western blotting results suggested that USP1 expression in CEM-C1 cells were higher than that in CEM-C7 cells, while GR expression in CEM-C1 was lower than that in CEM-C7 cells (Fig.1E-H, $P<0.05$ ). All findings indicated that USP1 were involved in the development of chemotherapy resistance in T-ALL.

\section{Downregulation of USP1 facilitated CEM-C1 cell chemosensitivity and apoptosis}

To evaluate the function of USP1, CEM-C1 cells were transfected with USP1 siRNA. And the results showed that the mRNA level of USP1 was markedly reduced compared with scramble group (Fig.2A, $P<$ 0.05). Then cells were treated with different concentrations of Dex to detect the sensitivity to chemotherapy. CCK-8 assay validated that cells transfection with USP1 siRNA could prominently ameliorate the sensitivity to Dex (Fig.2B and C, $P<0.05$ ). Transwell assay showed that downregulation of USP1 could reduce cell invasion (Fig.2D and E, $P<0.05$ ). Flow cytometry assay revealed that silence of USP1 could promote cell apoptosis (Fig.2F and G, $P<0.05$ ). In addition, USP1 silencing observably increased the levels of GR (Fig.2H and I, $P<0.05$ ). Taken together, downregulation of USP1 facilitated CEM-C1 cell sensitivity to Dex and cell apoptosis.

\section{USP1 interacted with Aurora B}

Abnormal Aurora B was reported to be associated with drug resistance of many human cancers[17]. Hence, we detected the expression of Aurora B in T-ALL patients. Our results revealed that Aurora B was upregulated in GC-sensitivity and GC-resistant T-ALL patients, and the level of Aurora B in bone marrow of patients with GC resistant T-ALL was higher (Fig.3 A, $P<0.05$ ). Pearson correlation test revealed that there was a positive correlation between USP1 and Aurora B in GC resistant patients (Fig.3B, $P<0.05$ ). Then we assessed whether USP1 could interact with Aurora B. Cells were transfected with FlagUSP1 and HA-Aurora B, and the binding of USP1 to Aurora B was verified by co-immunoprecipitation (Fig.3C and D, $P<0.05)$. Co-IP assay revealed that USP1 could bind to Aurora B (Fig. $3 \mathrm{E}, P<0.05)$. Western blotting 
assay revealed that downregulation of USP1 could reduce the level of Aurora B (Fig.3F and G, $P<0.05$ ). These results showed that USP1 interacted with Aurora B.

\section{USP1 regulated chemoresistance via Aurora B}

To investigate how USP1 and Aurora B affect chemoresistance, we used Aurora B inhibitors, barasertib, to explore the effects of USP1 and Aurora B in CEM-C1 cells. CEM-C1 cells were first treated with $5 \mu \mathrm{M}$ barasertib, and then transfected with pcDNA-USP1. The mRNA level of USP1 was upregulated after cells were transfected with pcDNA-USP1(Fig. 4A, $P<0.05$ ). Cells treatment with barasertib dramatically reduced the expression of Aurora $B$, while overexpression of USP1 could reverse the inhibitory effect of barasertib on Aurora B expression (Fig.4B and C, $P<0.05$ ). CCK-8, Transwell, flow cytometry and Western blot assays suggested that barasertib could increase the sensitivity of cells to Dex, suppress cell invasion, promote cell apoptosis and ameliorate the expression of GR (Fig.4C-E, $P<0.05)$. However, overexpression of USP1 recovered the promotion effects of barasertib on the sensitivity to Dex, cell apoptosis and GR expression in CEM-C1 cells and the inhibition of cell invasion (Fig.4C-E, $P<0.05$ ). All results demonstrated that USP1 promotes chemoresistance by interacting with Aurora B.

\section{ALKBH5 was upregulated in T-ALL patients and positively correlated with USP1}

ALKBH5 was reported to be the demethylase of m6A participated in tumorigenesis[18]. To explore the potential role of ALKBH5 in T-ALL, we analyzed the levels of ALKBH5 in T-ALL patients bone marrow. ALKBH5 expression was notably increased in GC-sensitivity group compared with normal group (Fig. 5A, $P<0.05)$. ALKBH5 level was higher in GC-resistance group than that in GC-sensitivity group $(P<0.05)$. Correlation analysis for USP1 and ALKBH5 was validated via StarBase and the results revealed that there was a positive correlation between USP1 and ALKBH5 (Fig.5B, $P<0.05$ ). Pearson correlation test showed that there was a positive correlation between the expression of USP1 and ALKBH5 in the bone marrow of GC-resistant patients (Fig.5C, $P<0.05$ ). ALKBH5 protein level was increased in CEM-C1 cells by comparison with CEM-C7 cells (Fig. 5D and E, $P<0.05$ ). Moreover, SRAMP, a prediction website of m6A modification sites, was indicated there were multiple m6A modification sites in USP1 mRNA sequence (Fig.5F). These results revealed that ALKBH5 was upregulated in T-ALL patients and positively correlated with USP1.

\section{Overexpression of USP1 counteracted the promotion of ALKBH5 silencing on Dex sensitivity and cell apoptosis}

To further explore the interrelation between ALKBH5 and USP1, a series of rescue experiments were performed. CEM-C1 cells were transfected with ALKBH5 siRNA alone or co-transfected with ALKBH5 siRNA and pCDNA-USP1. The levels of ALKBH5 and USP1 were downregulated after transfection with ALKBH5 siRNA. However, when CEM-C1 cells were co-transfected with ALKBH5 siRNA and pcDNA-USP1, ALKBH5 expression did not change, but USP1 level was reversed (Fig. 6A, $P<0.05$ ). Similarly, silence of ALKBH5 could decrease the level of Aurora $B$, while overexpression of USP1 recovered the expression of Aurora B (Fig. 6B and C, $P<0.05$ ). Additionally, downregulation of ALKBH5 ameliorated cell sensitivity to 
Dex, decrease the number of cell invasion, promoted cell apoptosis and increased GR expression, while overexpression of USP1 reversed all the promotion effects of ALKBH5 silence on cell sensitivity to Dex, apoptosis and GR expression and the inhibition effect of cell invasion (Fig. 6D-I, $P<0.05$ ). These findings suggested that overexpression of USP1 observably reversed the promotion effect of ALKBH5 silence on Dex sensitivity and cell apoptosis.

\section{Silence of USP1 prolonged the survival of mice with T-ALL}

Mice in control group lost weight and die on average after 22.5 days, while mice given with Dex could prolong the survival time (Fig. 7 A-C, $P<0.05$ ). Mice injected with sh-USP1 and given Dex could not only prolong the survival time but also maintain normal body weight $(P<0.05)$. Western blotting results revealed that mice injected with sh-USP1 and given Dex could reduce USP1 level and increase GR expression (Fig. 7 D-G, $P<0.05$ ).

\section{Discussion}

Ubiquitin modulates protein stability, localization or function through covalently binding to proteins. And ubiquitination participates in a plethora of cellular processes, including gene expression, cell apoptosis, DNA repair and others [19]. However, aberrant ubiquitin signaling is known to be a molecular causal link in certain cancer, immune system or cardiovascular diseases [20]. The inflammasomes promote the release of inflammatory cytokines by activating the ubiquitination of regulatory proteins, thereby regulating the innate immune response [21]. Autophagy receptor-bound ubiquitinated substrates may serve as a new pathway for selective autophagy [22]. Among these USPs, USP1 is one of the bestcharacterized USPs and plays a significant role in the regulation of cellular functions. Hyperactivation of USP1 contributed to breast cancer metastasis via deubiquitination and stabilization of KPNA2 expression [23]. USP1 mediated ovarian cancer cell resistance to platinum by regulating the stability of Snail [24]. However, the role of USP1 in T-ALL chemoresistance remains to explore. In our study, we found that USP1 was upregulated in bone marrow of GC-resistant patients compared with GC-sensitive patients. Downregulation of USP1 could ameliorate the sensitivity of CEM-C1 cells to Dex and promote cell apoptosis.

Aurora kinase B (Aurora-B) is a ubiquitously expressed serine/threonine kinase, which is also a key regulator of mitosis [17]. Recent studies have shown that overexpression of Aurora $B$ is closely related to chemoresistance. Aurora inhibitor repressed neuroblastoma resistance by reducing phosphorylation of aurora kinase substrate histone $\mathrm{H} 3$ [25]. Inhibition of Aurora kinase B attenuated chemoresistance in glioblastoma via a synergistic manner with temozolomide [26]. Elevated Aurora B expression contributed to chemoresistance and poor prognosis in breast cancer [27]. Therefore, Aurora B expression was highly in glucocorticoid-resistant T-ALL patients and cells.

Recently studies have shown that the ubiquitin-proteasome system participates in the regulation of a number of mitotic proteins activities and Aurora $B$ protein level is mainly via ubiquitination-mediated degradation [28]. USP14 associated with Aurora B and prevented Aurora B degradation to resist leukemic 
chemotherapy drugs-induced cell apoptosis [29]. USP7 regulated mitosis progression and taxane sensitivity by affecting the stability of Aurora kinase [30]. Hence, when CEM-C1 cells were treated with Aurora B inhibitors, CEM-C1 cell sensitivity to Dex increased, while overexpression of USP1 increased Aurora B levels, which in turn led to the cell resistance to Dex.

Glucocorticoids (GCs) are steroid hormones which exert their effects through glucocorticoid receptors (GRs) and participate in responses triggered by a variety of environmental and physiological stimuli, including inflammation, apoptosis [31]. Therefore, GCs are used to treat various malignancies, especially in the treatment of ALL. However, the generation of GC resistance hinders its efficacy. There are many mechanisms leading to GC resistance in leukemia patients, the most important of which is the abnormal expression of GR [32]. Decreased GR number or function may lead to GC resistance. AKT1 kinase acted as a major negative regulator of the NR3C1 glucocorticoid receptor protein activity, inducing glucocorticoid resistance in T-ALL [33]. CASP1 induced the division of the glucocorticoid receptor, reduced the glucocorticoid-induced transcriptional responses and increased glucocorticoid resistance in leukemia cells [34]. Therefore, our study found that downregulation of USP1 ameliorated the level of GR and increased cell sensitivity to Dex.

In this study, we exhibited a new role for USP1 in promoting chemoresistance via interacting with Aurora B. Elevated USP1 expression was associated with poor prognosis of patients with glucocorticoid resistance. ALKBH5 silence could suppress USP1 expression and reduce chemotherapeutic resistance. These results revealed that USP1 could be a novel biomarker for predicting chemoresistance in A-TLL.

\section{Declarations}

\section{Ethics approval and consent to participate}

This research was approved by the Medical Ethics Committee of the First Affiliated Hospital of Zhengzhou University. All patients had read and signed the informed consent.

\section{Acknowledgements}

No applicable.

\section{Funding}

This study was supported by the Youth Science Foundation of National Natural Science Foundation of China (No.81600097).

\section{Availability of data and materials}

The datasets used during the present study are available from the corresponding author on reasonable request.

\section{Authors' contributions}


LL designed this study and wrote the manuscript. HT performed the experimental work. LC provided the majority of statistical analysis as well as provided the figures and tables for the manuscript. HM and LS collected a large amount of data for the dataset. All authors read and approved the final manuscript.

\section{Patient consent for publication}

Consent for publication was obtained from each patient.

\section{Competing interests}

The authors declare that they have no competing interests.

\section{References}

1. Liu M, Liao H, Chen Y, Lin Z, Liu Y, Zhang X et al (2019) Treatment of human T-cell acute lymphoblastic leukemia cells with CFTR inhibitor CFTRinh-172. Leuk Res 86:106225.http://10.1016/j.leukres.2019.106225

2. Uscanga-Palomeque AC, Calvillo-Rodríguez KM, Gómez-Morales L, Lardé E, Denèfle T, CaballeroHernández D et al (2019) CD47 agonist peptide PKHB1 induces immunogenic cell death in T-cell acute lymphoblastic leukemia cells. Cancer Sci 110:256-268.http://10.1111/cas.13885

3. Qin T, Li B, Feng X, Fan S, Liu L, Liu D et al (2018) Abnormally elevated USP37 expression in breast cancer stem cells regulates stemness, epithelial-mesenchymal transition and cisplatin sensitivity. J Exp Clin Cancer Res 37:287.http://10.1186/s13046-018-0934-9

4. Chen J, Dexheimer TS, Ai Y, Liang Q, Villamil MA, Inglese J et al (2011) Selective and cell-active inhibitors of the USP1/ UAF1 deubiquitinase complex reverse cisplatin resistance in non-small cell lung cancer cells. Chem Biol 18:1390-1400.http://10.1016/j.chembiol.2011.08.014

5. Song Z, Li J, Zhang L, Deng J, Fang Z, Xiang X et al (2019) UCHL3 promotes pancreatic cancer progression and chemo-resistance through FOXM1 stabilization. Am J Cancer Res 9:1970-1981

6. He J, Lee HJ, Saha S, Ruan D, Guo H, Chan CH (2019) Inhibition of USP2 eliminates cancer stem cells and enhances TNBC responsiveness to chemotherapy. Cell Death Dis 10:285.http://10.1038/s41419019-1512-6

7. Wu C, Luo K, Zhao F, Yin P, Song Y, Deng M et al (2018) USP20 positively regulates tumorigenesis and chemoresistance through $\beta$-catenin stabilization. Cell Death Differ 25:18551869.http://10.1038/s41418-018-0138-z

8. Li L, Liu T, Li Y, Wu C, Luo K, Yin Y et al (2018) The deubiquitinase USP9X promotes tumor cell survival and confers chemoresistance through YAP1 stabilization. Oncogene 37:24222431.http://10.1038/s41388-018-0134-2

9. Chen Y, Peng C, Chen J, Chen D, Yang B, He B et al (2019) WTAP facilitates progression of hepatocellular carcinoma via m6A-HuR-dependent epigenetic silencing of ETS1. Mol Cancer 18:127.http://10.1186/s12943-019-1053-8

10. Ping XL, Sun BF, Wang L, Xiao W, Yang X, Wang WJ et al (2014) Mammalian WTAP is a regulatory subunit of the RNA N6-methyladenosine methyltransferase. Cell Res 24:177- 
189.http://10.1038/cr.2014.3

11. Li Q, Li X, Tang H, Jiang B, Dou Y, Gorospe M et al (2017) NSUN2-Mediated m5C Methylation and METTL3/METTL14-Mediated m6A Methylation Cooperatively Enhance p21 Translation. J Cell Biochem 118:2587-2598.http://10.1002/jcb.25957

12. Li T, Hu PS, Zuo Z, Lin JF, Li X, Wu QN et al (2019) METTL3 facilitates tumor progression via an m(6)A-IGF2BP2-dependent mechanism in colorectal carcinoma. Mol Cancer 18:112.http://10.1186/s12943-019-1038-7

13. Niu Y, Lin Z, Wan A, Chen H, Liang H, Sun L et al (2019) RNA N6-methyladenosine demethylase FTO promotes breast tumor progression through inhibiting BNIP3. Mol Cancer 18:46.http://10.1186/s12943-019-1004-4

14. Zhang S, Zhao BS, Zhou A, Lin K, Zheng S, Lu Z et al (2017) m(6)A Demethylase ALKBH5 Maintains Tumorigenicity of Glioblastoma Stem-like Cells by Sustaining FOXM1 Expression and Cell Proliferation Program. Cancer Cell 31:591-606 e596.http://10.1016/j.ccell.2017.02.013

15. Li J, Han Y, Zhang H, Qian Z, Jia W, Gao Y et al (2019) The m6A demethylase FTO promotes the growth of lung cancer cells by regulating the m6A level of USP7 mRNA. Biochem Biophys Res Commun 512:479-485.http://10.1016/j.bbrc.2019.03.093

16. Li Z, Weng H, Su R, Weng X, Zuo Z, Li C et al (2017) FTO Plays an Oncogenic Role in Acute Myeloid Leukemia as a N(6)-Methyladenosine RNA Demethylase. Cancer Cell 31:127141.http://10.1016/j.ccell.2016.11.017

17. Yu J, Zhou J, Xu F, Bai W, Zhang W (2018) High expression of Aurora-B is correlated with poor prognosis and drug resistance in non-small cell lung cancer. The International journal of biological markers 33:215-221.http://10.1177/1724600817753098

18. Zhang C, Samanta D, Lu H, Bullen JW, Zhang H, Chen I et al (2016) Hypoxia induces the breast cancer stem cell phenotype by HIF-dependent and ALKBH5-mediated m $\triangle A$-demethylation of NANOG mRNA. Proc Natl Acad Sci U S A 113:E2047-2056.http://10.1073/pnas.1602883113

19. García-Santisteban I, Peters GJ, Giovannetti E, Rodríguez JA (2013) USP1 deubiquitinase: cellular functions, regulatory mechanisms and emerging potential as target in cancer therapy. Mol Cancer 12:91.http://10.1186/1476-4598-12-91

20. Faktor J, Pjechová M, Hernychová L, Vojtěšek B (2019) Protein Ubiquitination Research in Oncology. Klin Onkol 32:56-64.http://10.14735/amko20193S

21. Bednash JS, Mallampalli RK (2016) Regulation of inflammasomes by ubiquitination. Cell Mol Immunol 13:722-728.http://10.1038/cmi.2016.15

22. Shaid S, Brandts $\mathrm{CH}$, Serve H, Dikic I (2013) Ubiquitination and selective autophagy. Cell Death Differ 20:21-30.http://10.1038/cdd.2012.72

23. Ma A, Tang M, Zhang L, Wang B, Yang Z, Liu Y et al (2019) USP1 inhibition destabilizes KPNA2 and suppresses breast cancer metastasis. Oncogene 38:2405-2419.http://10.1038/s41388-018-0590-8

24. Sonego M, Pellarin I, Costa A, Vinciguerra GLR, Coan M, Kraut A et al (2019) USP1 links platinum resistance to cancer cell dissemination by regulating Snail stability. Sci Adv 
5:eaav3235.http://10.1126/sciadv.aav3235

25. Michaelis M, Selt F, Rothweiler F, Löschmann N, Nüsse B, Dirks WG et al (2014) Aurora kinases as targets in drug-resistant neuroblastoma cells. PLoS One 9:e108758.http://10.1371/journal.pone.0108758

26. Alafate W, Wang M, Zuo J, Wu W, Sun L, Liu C et al (2019) Targeting Aurora kinase B attenuates chemoresistance in glioblastoma via a synergistic manner with temozolomide. Pathol Res Pract 215:152617.http://10.1016/j.prp.2019.152617

27. Zhang Y, Jiang C, Li H, Lv F, Li X, Qian X et al (2015) Elevated Aurora B expression contributes to chemoresistance and poor prognosis in breast cancer. Int J Clin Exp Pathol 8:751-757

28. Park J, Song EJ (2018) Deubiquitinase USP35 as a novel mitotic regulator via maintenance of Aurora B stability. BMB Rep 51:261-262.http://10.5483/bmbrep.2018.51.6.110

29. Song C, Ma R, Yang X, Pang S (2017) The Deubiquitinating Enzyme USP14 Regulates Leukemic Chemotherapy Drugs-Induced Cell Apoptosis by Suppressing Ubiquitination of Aurora Kinase B. Cell Physiol Biochem 42:965-973.http://10.1159/000478679

30. Giovinazzi S, Morozov VM, Summers MK, Reinhold WC, Ishov AM (2013) USP7 and Daxx regulate mitosis progression and taxane sensitivity by affecting stability of Aurora-A kinase. Cell Death Differ 20:721-731.http://10.1038/cdd.2012.169

31. Isikbay M, Otto K, Kregel S, Kach J, Cai Y, Vander Griend DJ et al (2014) Glucocorticoid receptor activity contributes to resistance to androgen-targeted therapy in prostate cancer. Horm Cancer 5:7289.http://10.1007/s12672-014-0173-2

32. Rodriguez JM, Monsalves-Alvarez M, Henriquez S, Llanos MN, Troncoso R (2016) Glucocorticoid resistance in chronic diseases. Steroids 115:182-192.http://10.1016/j.steroids.2016.09.010

33. Piovan E, Yu J, Tosello V, Herranz D, Ambesi-Impiombato A, Da Silva AC et al (2013) Direct reversal of glucocorticoid resistance by AKT inhibition in acute lymphoblastic leukemia. Cancer Cell 24:766776.http://10.1016/j.ccr.2013.10.022

34. Paugh SW, Bonten EJ, Savic D, Ramsey LB, Thierfelder WE, Gurung P et al (2015) NALP3 inflammasome upregulation and CASP1 cleavage of the glucocorticoid receptor cause glucocorticoid resistance in leukemia cells. Nat Genet 47:607-614.http://10.1038/ng.3283

\section{Figures}


A
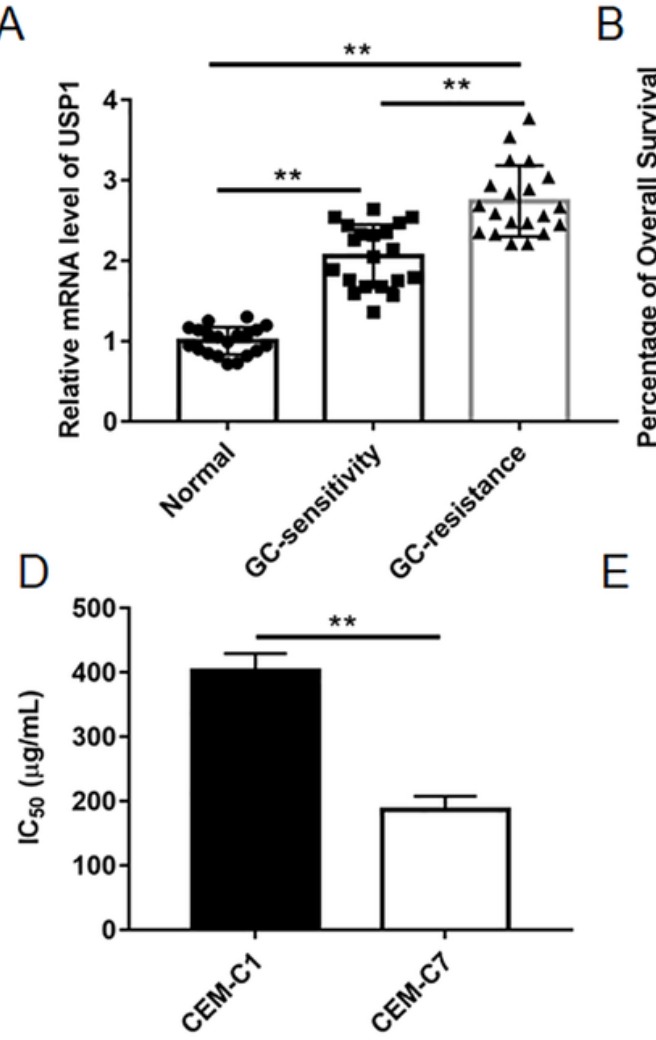

G

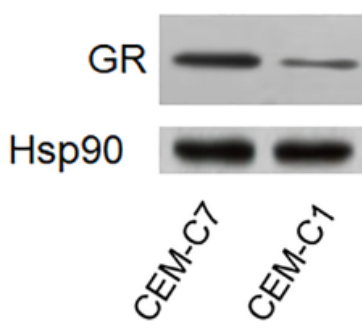

$\mathrm{B}$

E

$\mathrm{H}$
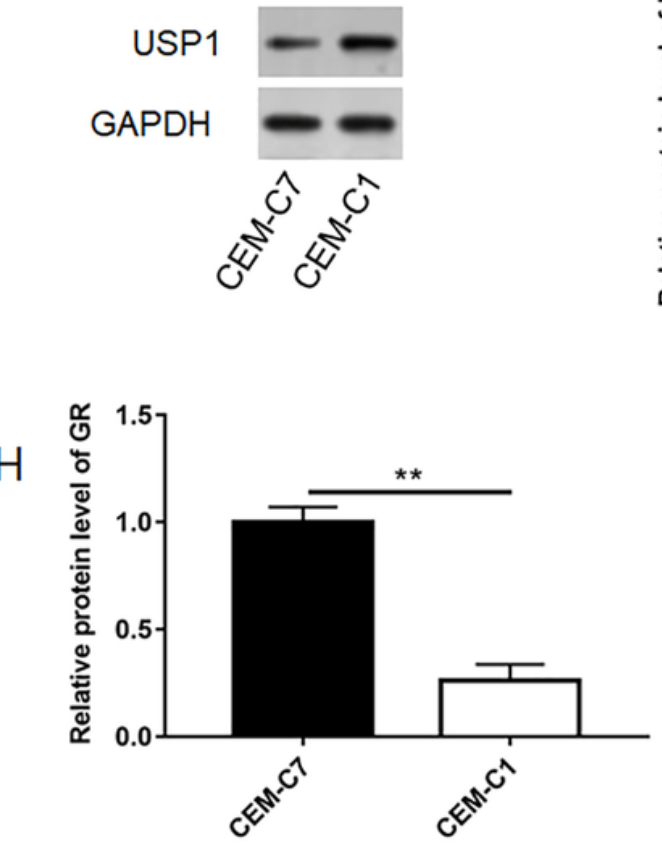

C

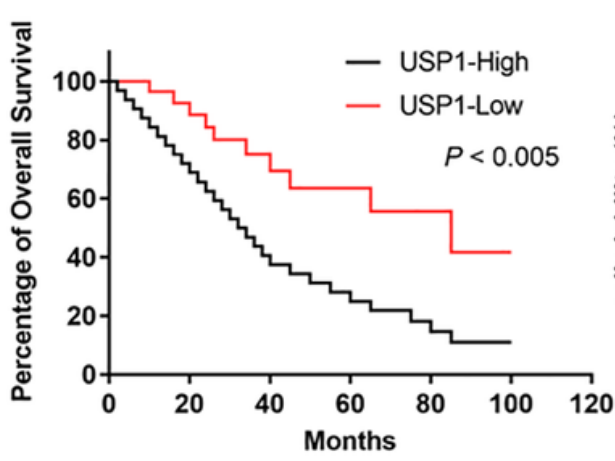

$\mathrm{F}$
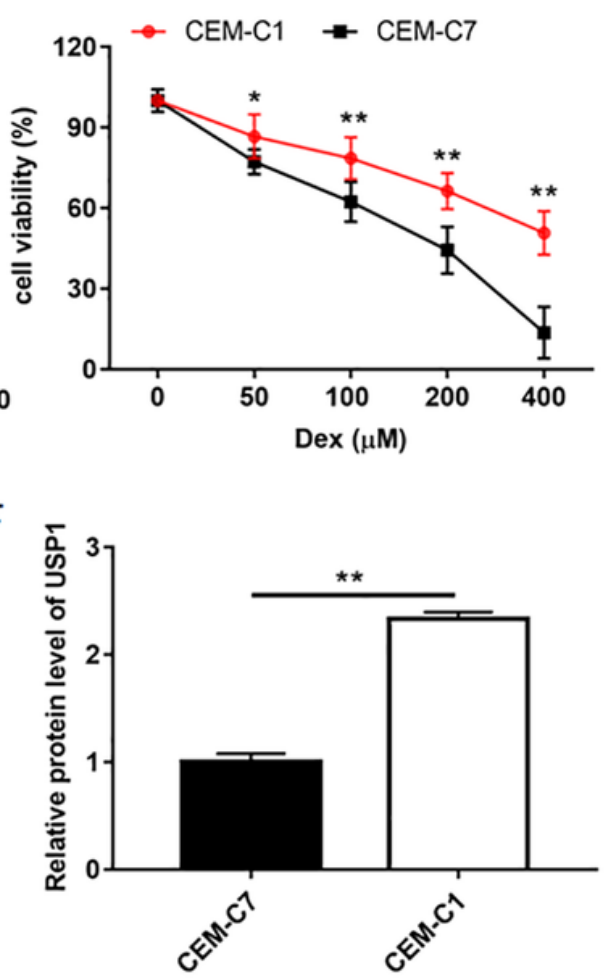

\section{Figure 1}

USP1 was upregulated in the bone marrow of T-ALL patients. (A) The MRNA level of USP1 was detected using RT-qPCR. (B) The Kaplan-Meier plot indicated the overall survival in T-ALL patients grouped by high or low level of USP1. (C) Cell viability was conducted after CEM-C7 or CEM-C1 cells were treated with different concentrations of Dex. (D) IC50 of Dex in CEM-C7 and CEM-C1. (E-H) Western blot assay was performed to analyze the levels of USP1 and GR. ${ }^{*} \mathrm{P}<0.05$ compared with normal group, GC-sensitivity group or CEM-C7 group. 
A

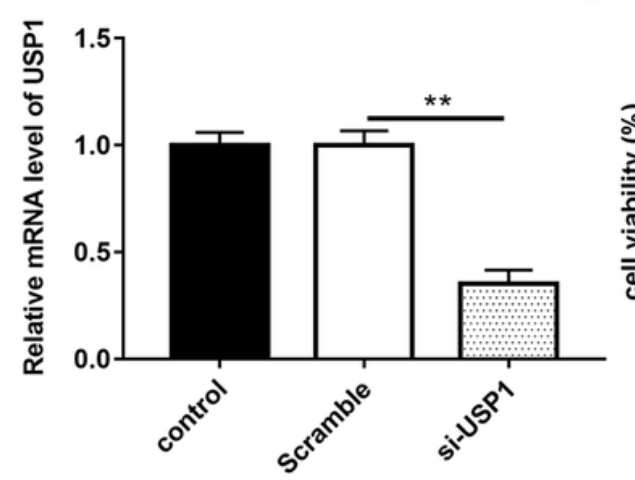

D

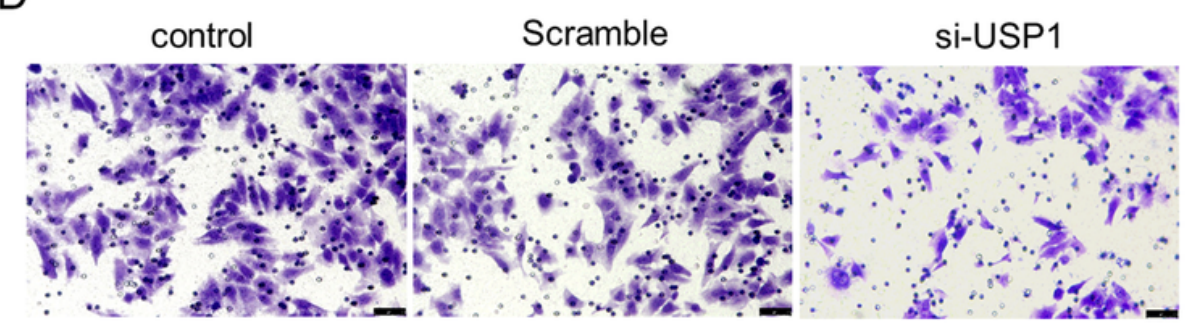

F
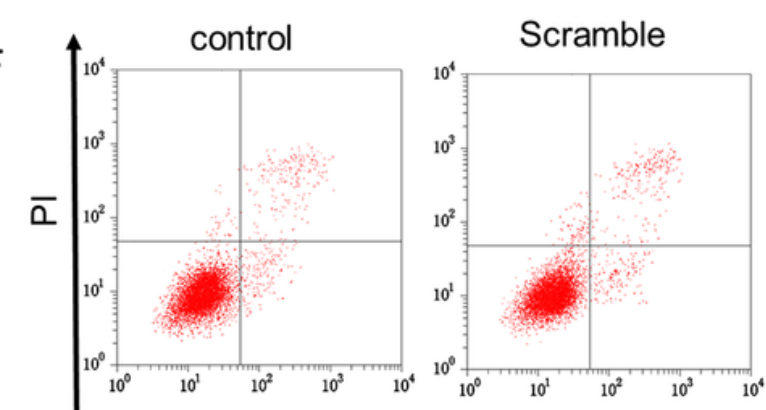

Annexin V FITC

$\mathrm{H}$

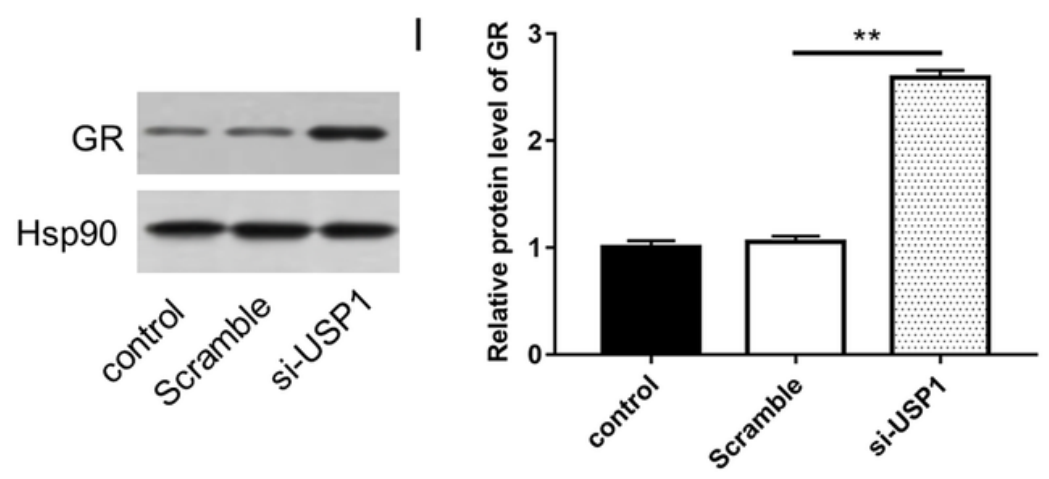

C
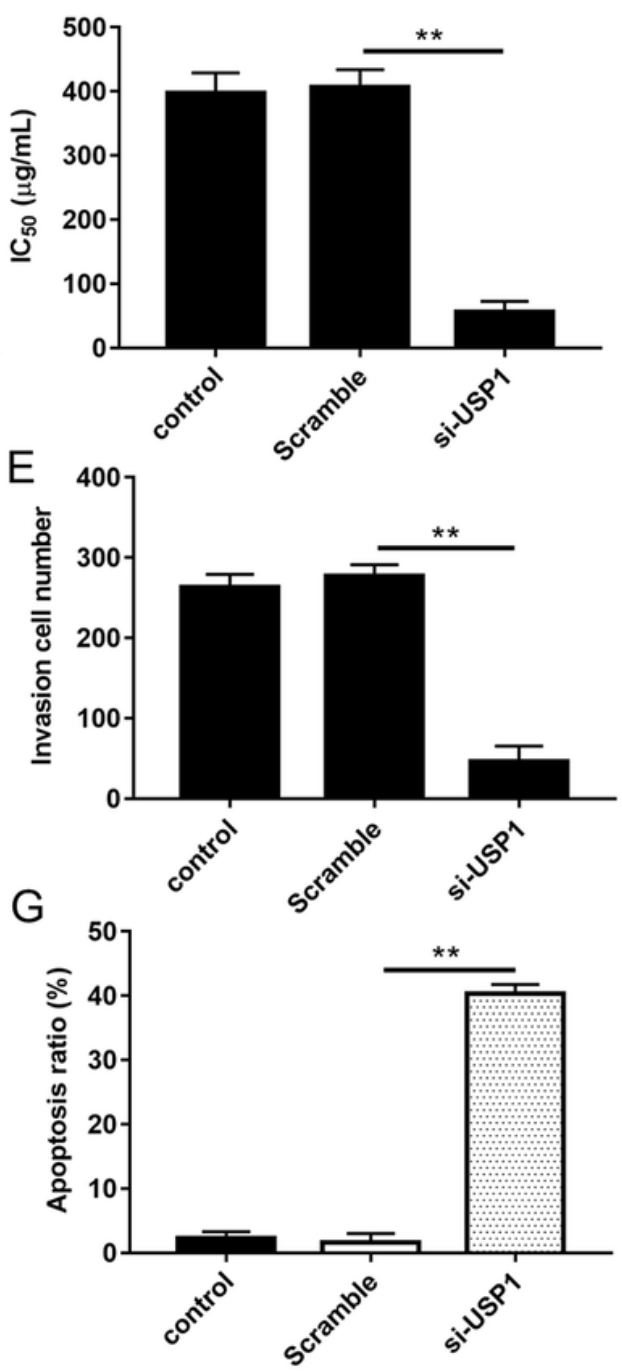

\section{Figure 2}

Downregulation of USP1 facilitated CEM-C1 cell chemosensitivity and cell apoptosis. (A) RT-qPCR was conducted to detect the level of USP1 after cells were transfected with USP1 siRNA. (B) Cell viability was evaluated by CCK-8 assay. (C) IC50 of Dex in CEM-C1 cells after transfection with USP1 siRNA. (D-E) Cell invasion was analyzed by using Transwell. (F-G) Flow cytometry assay was carried out to analyze cell apoptosis. $(\mathrm{H}-\mathrm{I})$ The level of GR was detected by western blot assay after cells were transfected with USP1 siRNA. *P $<0.05$ compared with control group and scramble group. 


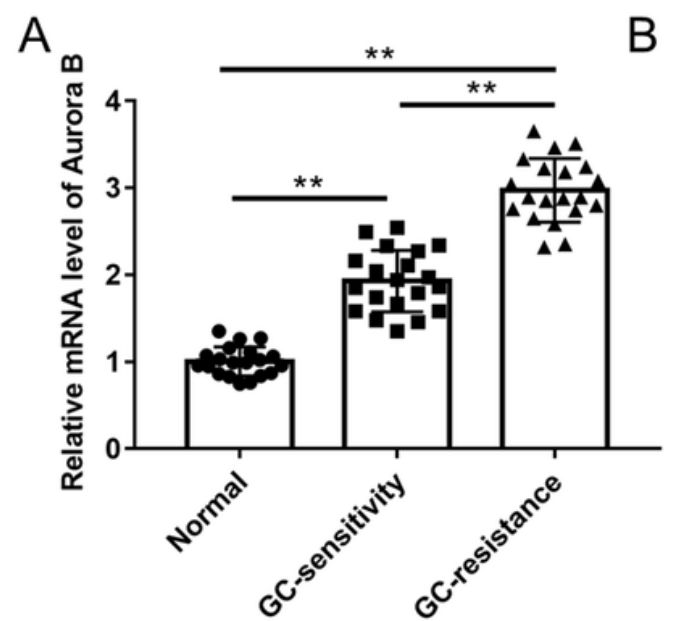

D

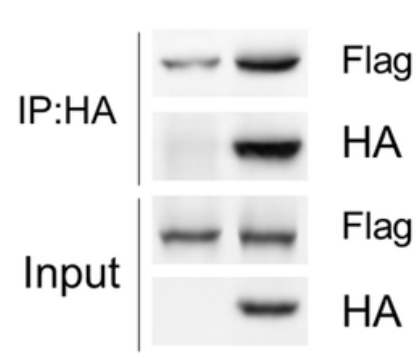

HA-Aurora B - +

Flag-USP1 ++

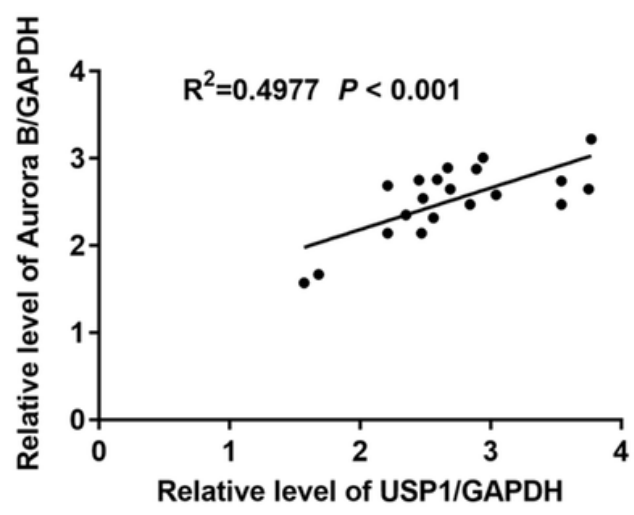

$E$

USP1

Aurora B

Input IgG Aurora B

IP

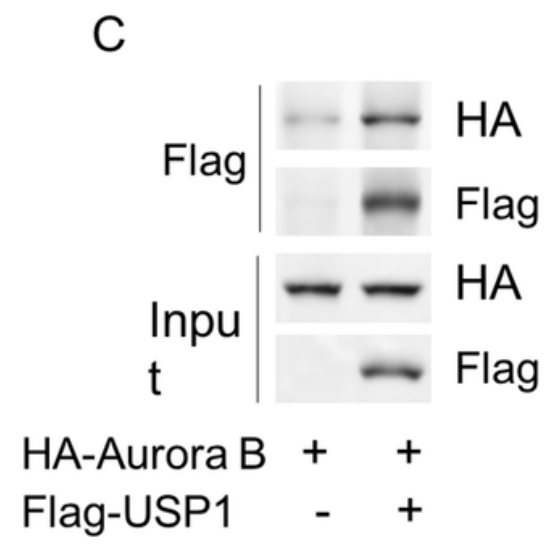

F

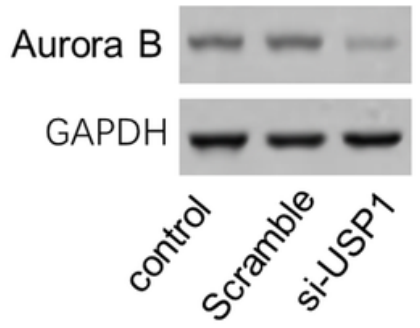

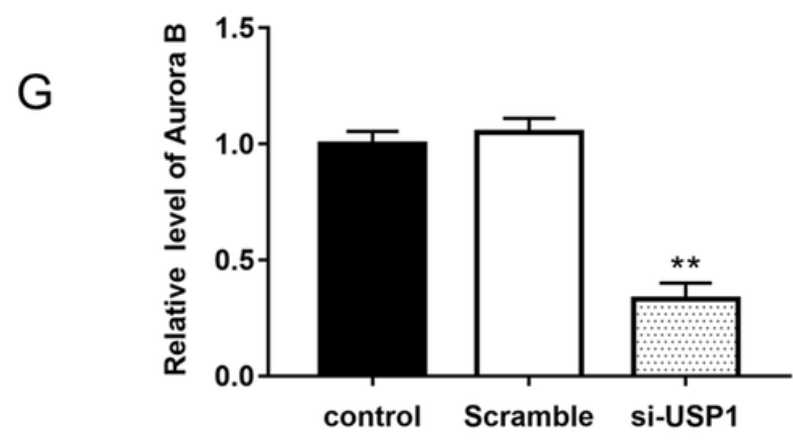

\section{Figure 3}

USP1 interacted with Aurora B. (A) The mRNA level of Aurora B was detected using RT-qPCR. (B) Correlation between USP1 and Aurora B was analyzed with Pearson Correlation analysis. (C) Cells were transfected with HA-Aurora B alone or in combination with Flag-USP1. Immunoprecipitation was performed to detect the interaction between Flag-USP1 and HA-Aurora B after immunoprecipitation with an anti-Flag antibody. (D) Cells were transfected with Flag-USP35 alone or in combination with HA-Aurora B. The interaction between Flag-USP35 and HA-Aurora B was detected by immunoblotting after immunoprecipitation with an anti-HA antibody. (E) The interaction between endogenous USP35 and Aurora B was detected by immunoblotting after immunoprecipitation with an anti-Aurora B antibody. (F$G$ ) Western blot assay was performed to analyze the level of Aurora B after cells were transfected with USP1 siRNA. *P $<0.05$ compared with IgG group or scramble group. 


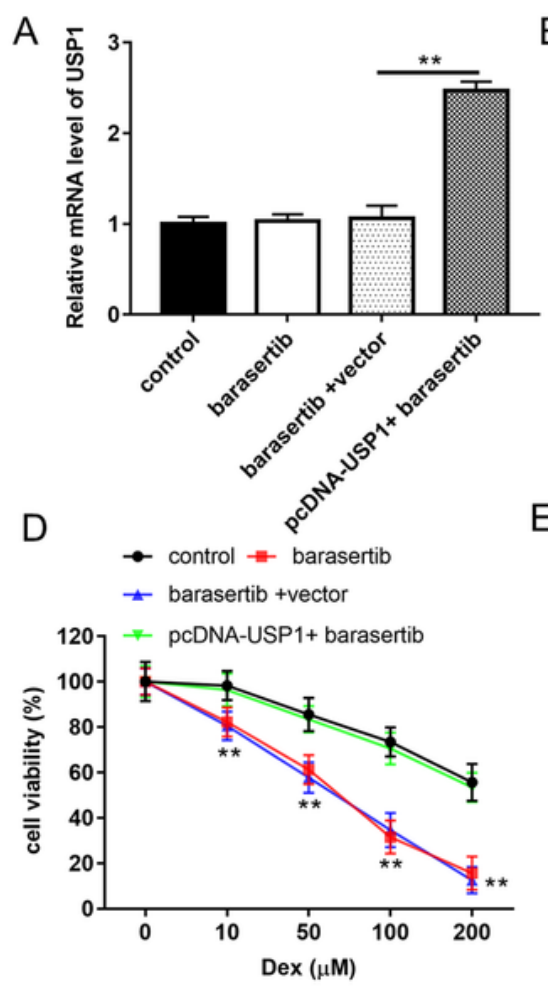

B

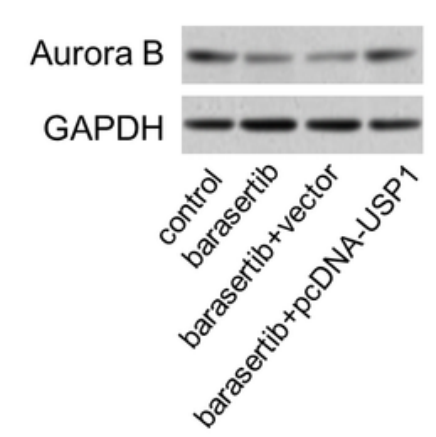

E

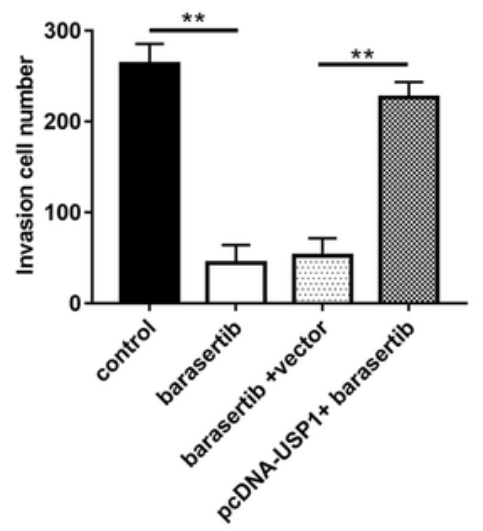

C

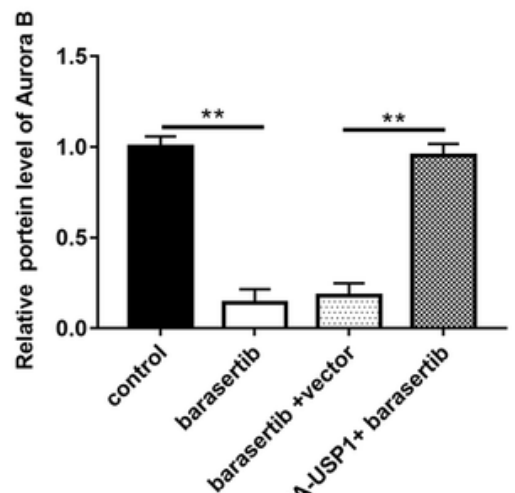

F

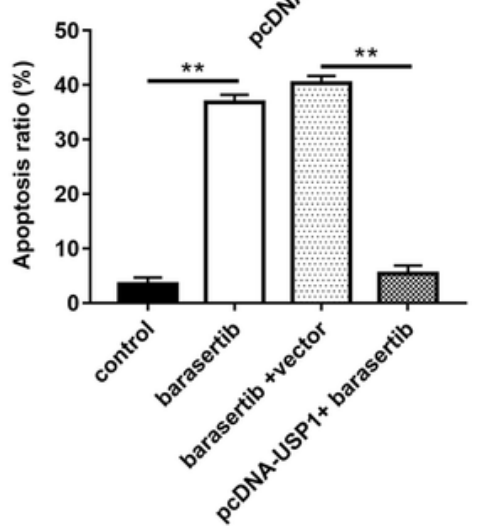

G

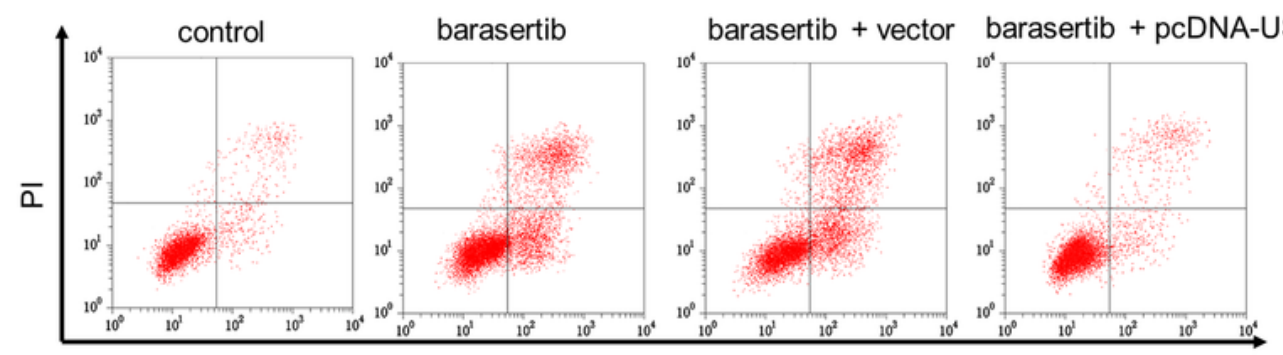

Annexin V FITC

$\mathrm{H}$
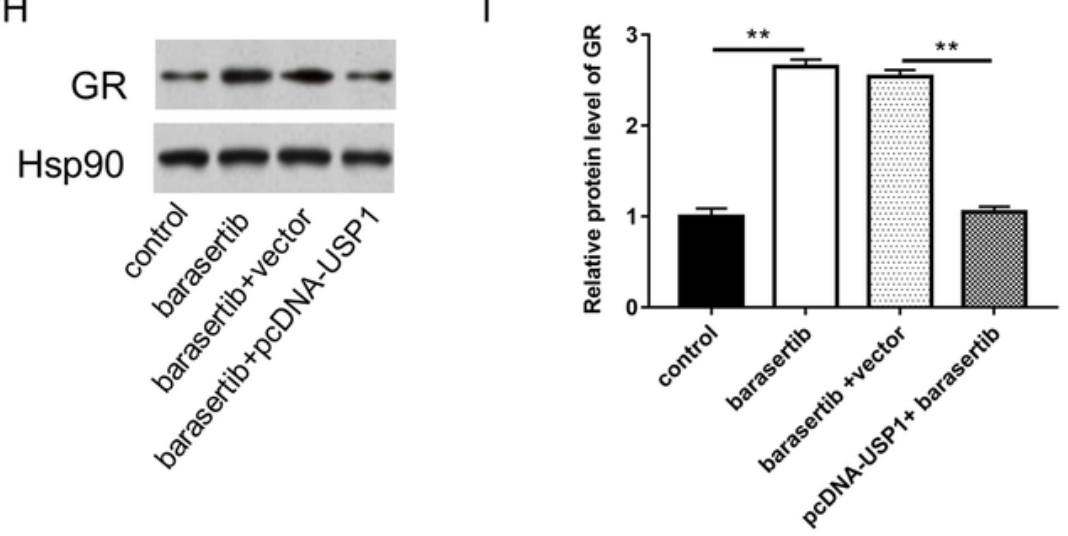

\section{Figure 4}

USP1 regulated chemoresistance via Aurora B. (A) The level of USP1 was detected using qPCR. (B-C) The protein level of Aurora B was analyzed using western blot assay. (D) Cell viability was evaluated by CCK-8 assay. (E) Cell invasion was analyzed by using Transwell. (F-G) Flow cytometry assay was carried out to analyze cell apoptosis. $(\mathrm{H}-\mathrm{I})$ The levels of $\mathrm{GR}$ were detected by western blot assay. ${ }^{*} \mathrm{P}<0.05$ compared with control group or barasertib with vector group. 
A

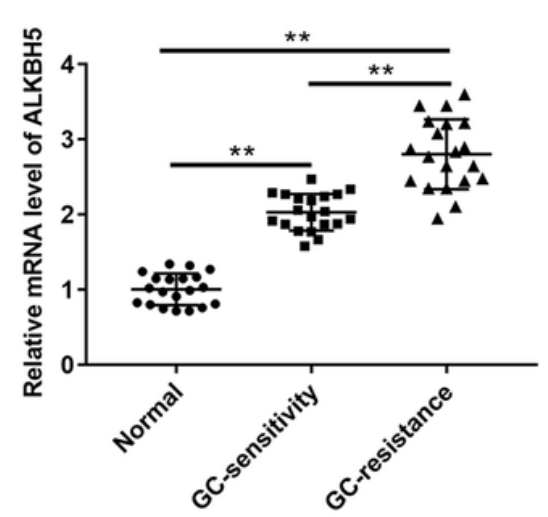

D

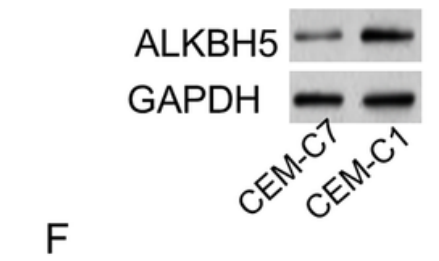

B

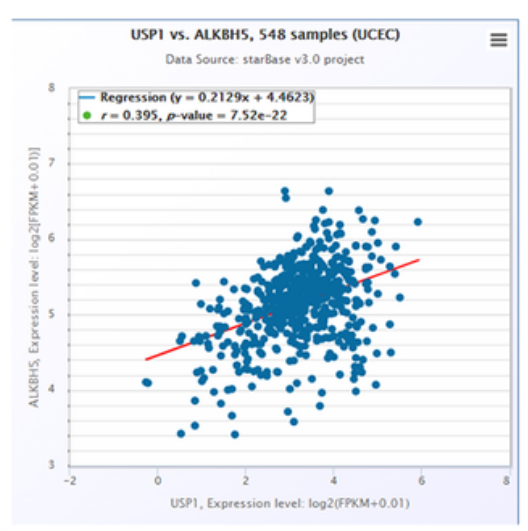

E

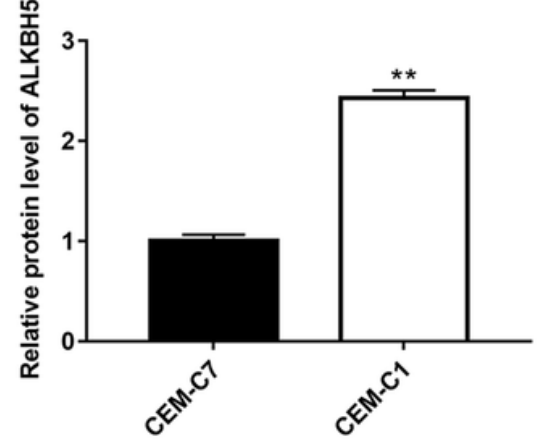

C

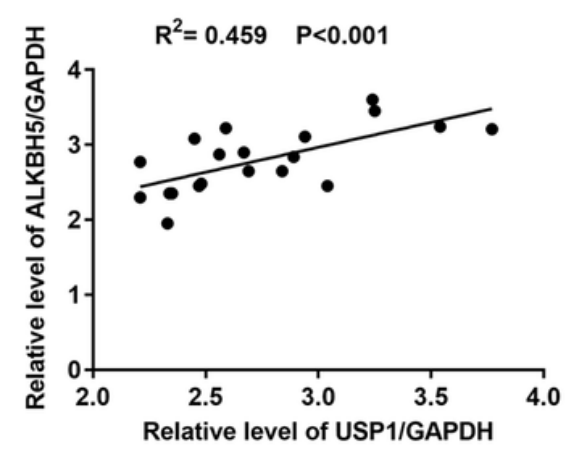

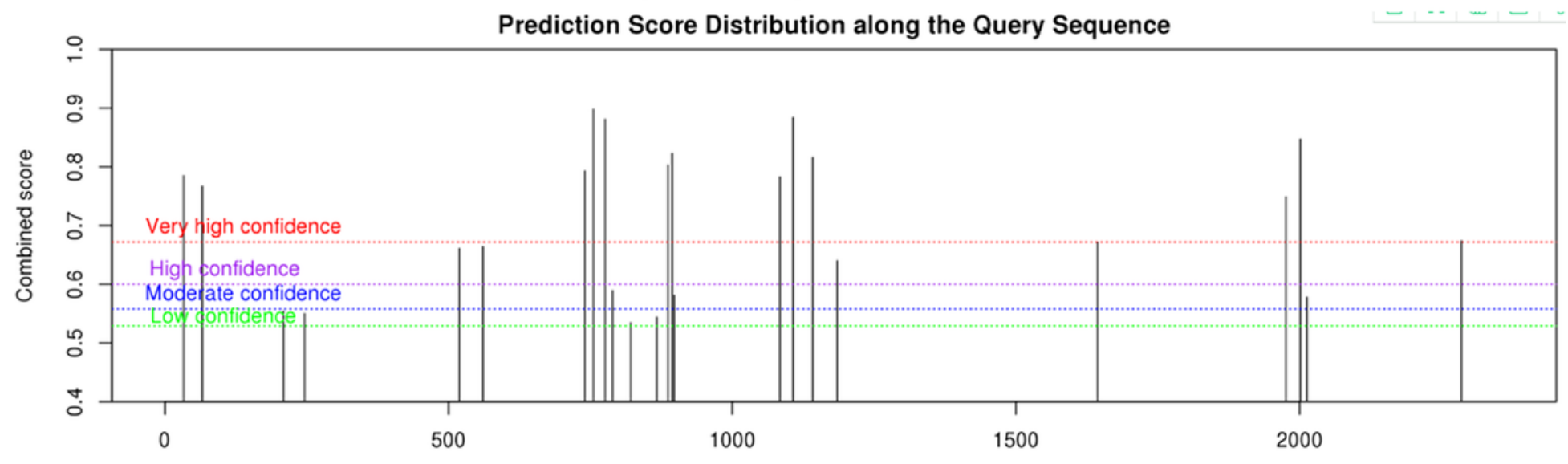

Figure 5

ALKBH5 was upregulated in T-ALL patients and positively correlated with USP1. (A) The mRNA level of ALKBH5 was detected by RT-qPCR. (B) Correlation analysis for USP1 and ALKBH5 was validated via StarBase. (C) Correlation between USP1 and ALKBH5 was analyzed with Pearson Correlation analysis. (D) Western blot assay was used to analyze the level of ALKBH5 in CEM-C7 and CEM-C1 cells. (E) m6A modification sites of USP1 was predicted by SRAMP. ${ }^{*} \mathrm{P}<0.05$ compared with normal group, GCsensitivity group or CEM-C7 group. 

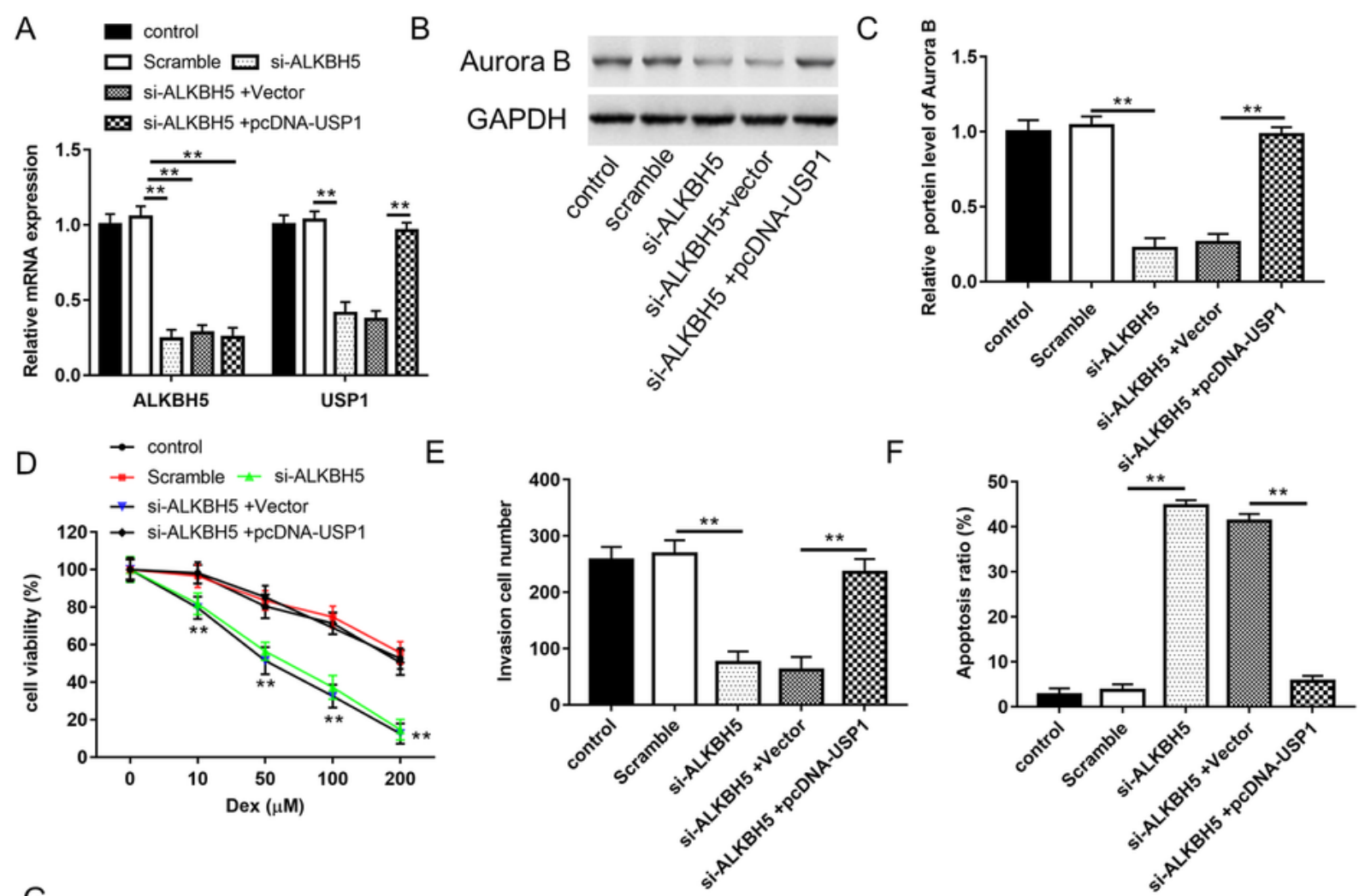

G

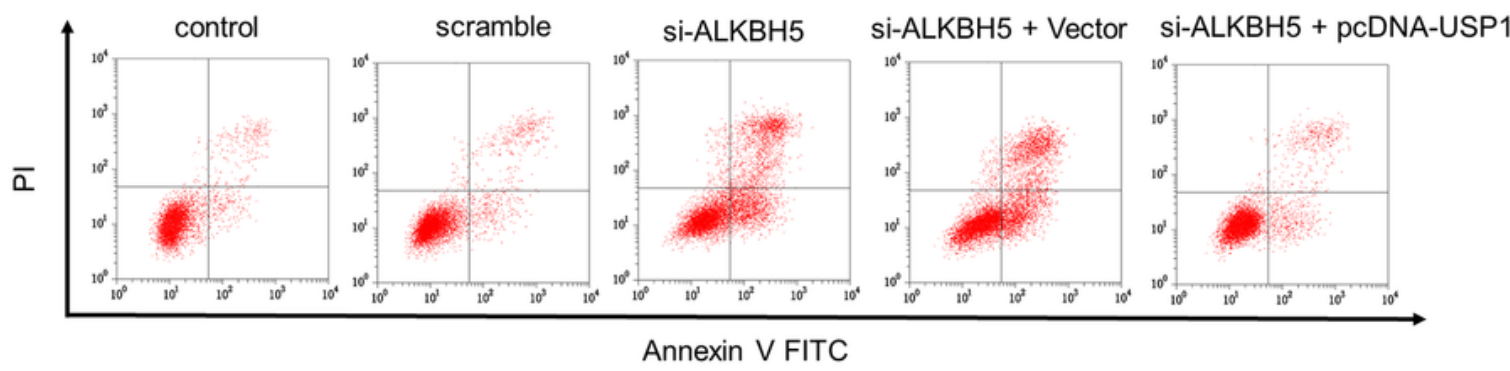

$\mathrm{H}$
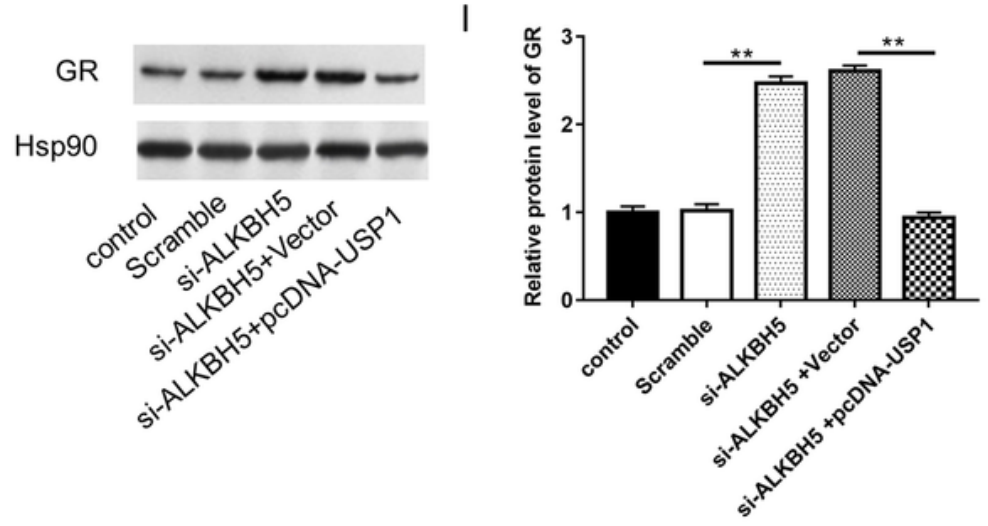

\section{Figure 6}

Overexpression of USP1 counteracted the promotion of ALKBH5 silence on Dex sensitivity and cell apoptosis. (A-B) The levels of ALKBH5, USP1 and Aurora B were analyzed by RT-qPCR after cells were transfected with ALKBH5 siRNA or co-transfection with ALKBH5 siRNA and pcDNA-USP1. (C) Cell viability was evaluated by CCK-8 assay. (D) Cell invasion was analyzed by using Transwell. (F-G) Flow cytometry 

assay. ${ }^{*} \mathrm{P}<0.05$ compared with control group, scramble group, ALKBH5 siRNA group and Vector group.

A
B

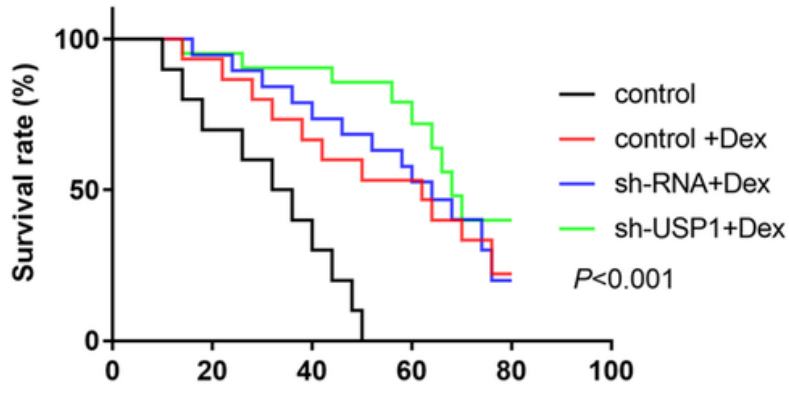

C

CEM-C1

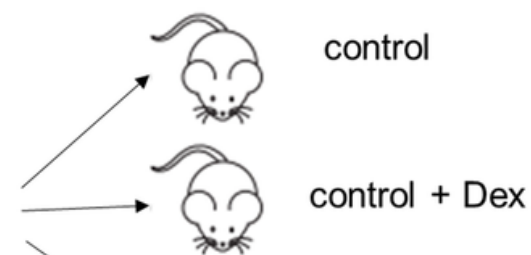

sh-RNA +Dex

sh-USP1 +Dex
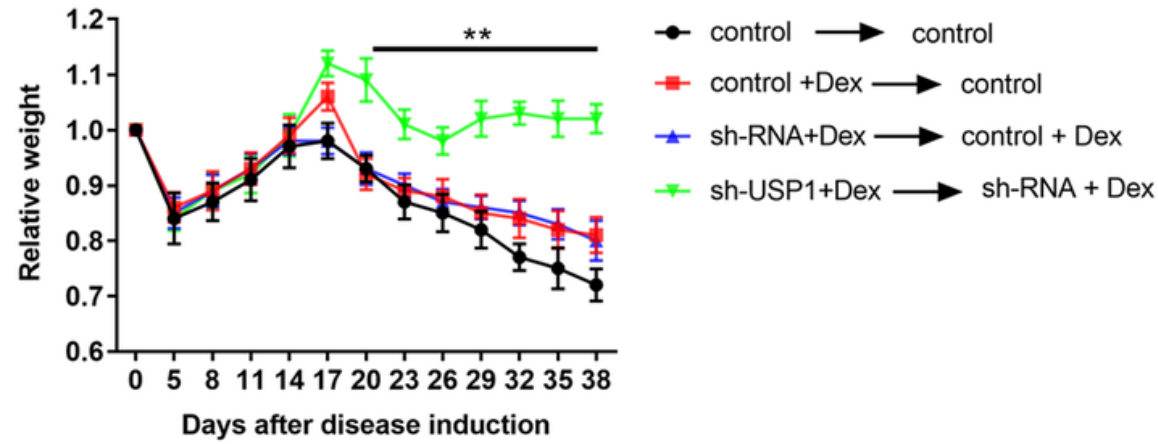

E

F
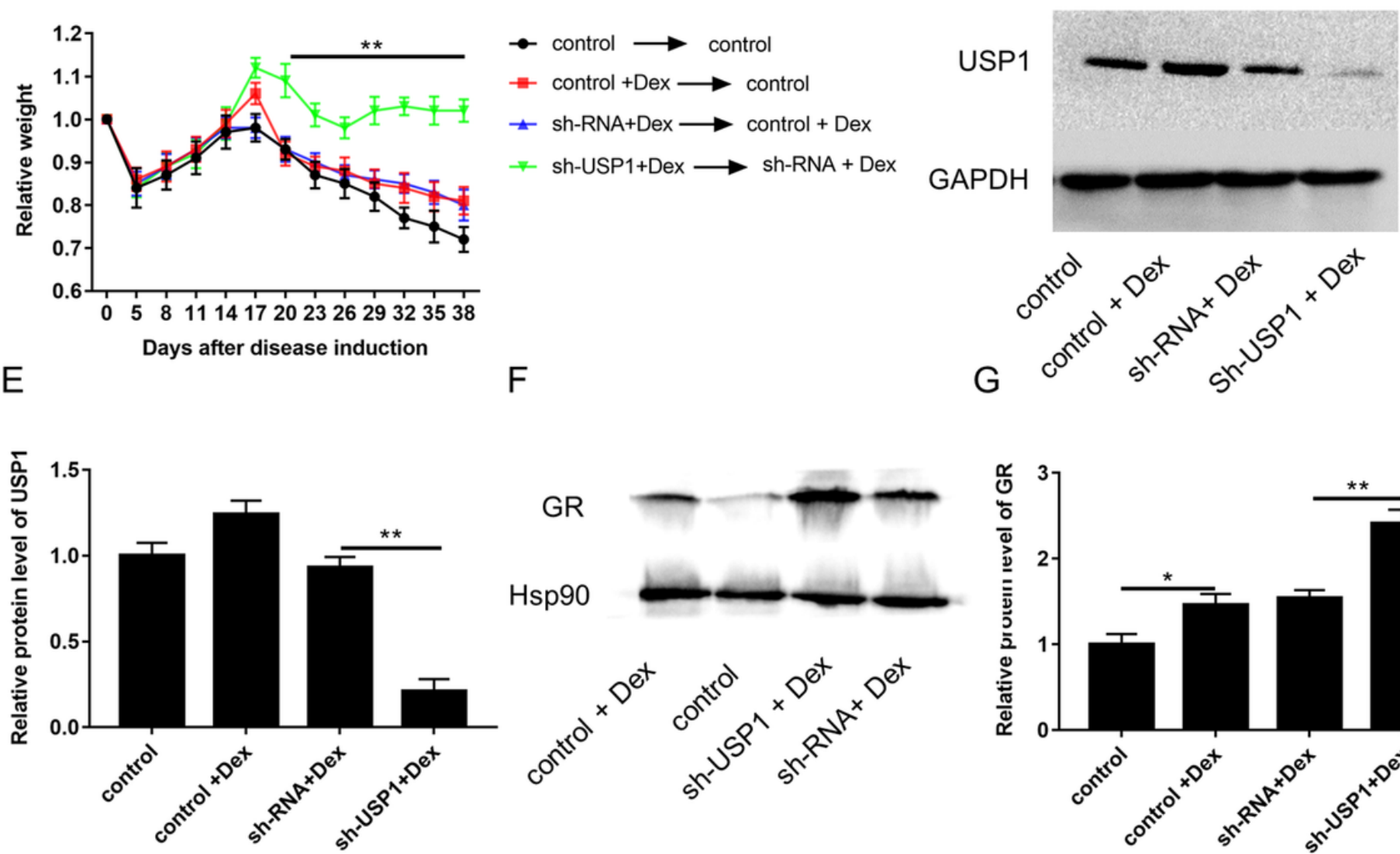

GR

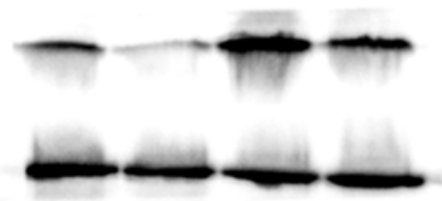

Hsp90

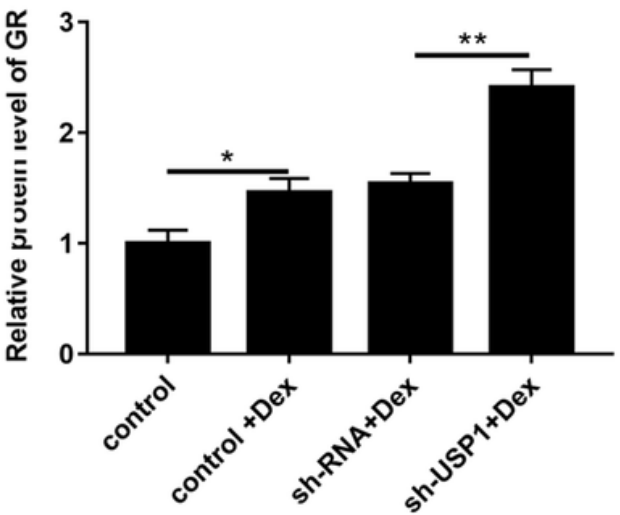

Figure 7

Silence of USP1 prolonged the survival of mice with T-ALL. (A) After CEM-C1 cells were injected into mice, mice were given with $8 \mathrm{mg} / \mathrm{kg}$ Dex only or simultaneously injected with $2 \mathrm{mg} / \mathrm{Kg}$ sh-RNA or sh-USP1. (B) Survival of mice in each group. (C) Relative weight of mice. (D-G) The protein levels of USP1 and GR in each group were detected by using Western blotting. ${ }^{*} \mathrm{P}<0.05$ compared with control and sh-RNA + Dex group. 